Regular Article

\title{
The value of tomographic ventilation/perfusion scintigraphy (V/PSPECT) for follow-up and prediction of recurrence in pulmonary embolism
}

\author{
Alaa Alhadad ${ }^{\mathrm{a}, *}$, Massimo Miniati $^{\mathrm{b}}$, Hussein Alhadad ${ }^{\mathrm{a}}$, Anders Gottsäter ${ }^{\mathrm{a}}$, Marika Bajc ${ }^{\mathrm{c}}$ \\ a Department of Vascular Diseases, Skåne University Hospital, S-205 02 Malmö, Sweden \\ ${ }^{b}$ Department of Medical and Surgical Critical Care, University Hospital Florence, Italy \\ c Department of Clinical Physiology, Skåne University Hospital, S-221 85 Lund, Sweden
}

\section{A R T I C L E I N F O}

\section{Article history:}

Received 15 July 2012

Received in revised form 27 August 2012

Accepted 3 September 2012

Available online $\mathrm{xxxx}$

\section{Keywords:}

pulmonary embolism

lung scintigraphy

V/P SPECT

pulmonary embolism recurrence

\begin{abstract}
A B S T R A C T
Background: Pulmonary embolism (PE) is diagnosed with imaging techniques such as ventilation/perfusion $(\mathrm{V} / \mathrm{P})$ lung scintigraphy or multidetector computed tomography of the pulmonary arteries (MDCT). Lung scintigraphy can be performed with planar (V/P PLANAR) and tomographic (V/P SPECT) techniques. V/P SPECT has higher sensitivity and specificity than V/P PLANAR. As nephrotoxic contrast media are not used during V/P SPECT, examinations can be repeated for evaluation of resolution of perfusion defects after PE. However, the value of residual perfusion defects identified using V/P SPECT for the prediction of recurrent PE has not been thoroughly evaluated.

Material and methods: We evaluated resolution and recurrence of PE in 227patients (mean age $63 \pm 17$ years, 134[59\%] women) with PE undergoing $\geq 2$ SPECT examinations in 2005-2007. PE was defined as minor $(<20 \%$ perfusion defect on SPECT, $\mathrm{n}=86)$, medium $(20-50 \%$ perfusion defect on SPECT, $\mathrm{n}=99)$, or major ( $>50 \%$ perfusion defect on SPECT, $n=42$ ).

Results: At second V/P SPECT examination, complete resolution of perfusion defects had occurred in 45 (52\%) patients with minor PE after $8.2 \pm 7.4$ months, in 29 (29\%) of patients with medium PE after $6.2 \pm 5.9$ months, and in $2(5 \%)$ of patients with major PE after $6.5 \pm 0.7$ months. During $47 \pm 24$ months of follow up, 37(16 \%) patients suffered recurrent PE. Of these 37, 34 (92\%) showed residual perfusion defects at the second V/P SPECT examination. Recurrence of PE was also predicted by advanced age and female gender. However, in multivariate regression analysis, recurrence was only predicted by age $(p=0.0013)$ and residual perfusion defect on V/P SPECT ( $\mathrm{p}=0.0039$ ).

Conclusion: In conclusion, complete resolution of PE was common in patients with minor PE, whereas residual perfusion defects were widespread in patients with medium and major PE. PE patients identified with persistent perfusion defects at follow-up SPECT have a high risk of PE recurrence.
\end{abstract}

(c) 2012 Elsevier Ltd. All rights reserved.

\section{Introduction}

Pulmonary embolism (PE) is a common and potentially fatal disorder in which clinical symptoms and laboratory findings are non specific. A firm diagnosis of PE can only be made by using imaging techniques such as ventilation/perfusion (V/P) lung scintigraphy or multidetector computed tomography of the pulmonary arteries (MDCT). Scintigraphy with tomographic (V/P SPECT) technique has higher sensitivity and specificity than with planar (V/P PLANAR) technique [1-3].The strength of V/P SPECT is based on its sensitivity, specificity, and applicability to all patients, regardless of age, kidney function or any other diseases [4]. Moreover, V/P SPECT confers a lower and predictable radiation burden [5] particularly regarding absorbed breast doses [6,7]. Both V/P SPECT and MDCT not only allow diagnosis of PE, but also enable visualisation of other cardiopulmonary diseases [4].

\footnotetext{
* Corresponding author. Tel.: + 4640 331000; fax: +46 40338097

E-mail address: alaa.alhadad@gmail.com (A. Alhadad).
}

However, the resolution of perfusion defects after a PE diagnosis is insufficiently studied. Current recommendations for treatment duration after PE $[8,9]$ are also partly based on insufficient evidence. Patients are usually treated with anticoagulants for 6 months, a therapy conferringrisk of serious bleeding complications and inconvenience because of the need for regular monitoring $[9,10]$. More individual tailoring of anticoagulant treatment duration would be extremely valuable in this patient group.

It would therefore be of great clinical value to have a safe investigational method which could be used in as many patients as possible, not only for the diagnosis of PE, but also in routine follow-up after diagnosis to visualise resolution of perfusion defects and to help in the prediction of PE recurrence and the determination of treatment duration. Such follow-up is not feasible with MDCT, however, as the method exposes patients to substantial amount of radiation [11,12]and should therefore not be used for routine follow-up. Moreover, MDCT does not allow quantification of PE extension and has very low sensitivity in the diagnosis of chronic PE [13-15].V/P SPECT, on the other hand offers these possibilities. 
Table 1

Baseline data in 227 patients with pulmonary embolism (PE) undergoing 2 examinations with ventilation / perfusion (V/P) lung scintigraphy with tomographic (SPECT) technique, and in subgroups of patients with minor $(<20 \%$ perfusion defect on SPECT), medium (20-50\% perfusion defect on SPECT), or major ( $>50 \%$ perfusion defect on SPECT, ) PE.Mean \pm SD or $n(\%)$.

\begin{tabular}{lllll}
\hline & $\begin{array}{l}\text { All patients, } \\
\mathrm{n}=227\end{array}$ & $\begin{array}{l}\text { Minor PE, } \\
\mathrm{n}=86\end{array}$ & $\begin{array}{l}\text { Medium PE, } \\
\mathrm{n}=99\end{array}$ & $\begin{array}{l}\text { Major PE, } \\
\mathrm{n}=42\end{array}$ \\
\hline Age (years) & $63 \pm 17$ & $59 \pm 20$ & $64 \pm 17$ & $70 \pm 11$ \\
Gender M/F & $94 / 134$ & $33 / 53$ & $37 / 62$ & $24 / 18$ \\
Prior VTE (n[\%]) & $50(22)$ & $10(12)$ & $31(31)$ & $9(21)$ \\
Malignancy(n[\%]) & $51(22)$ & $20(23)$ & $20(20)$ & $11(26)$ \\
$\begin{array}{c}\text { Blood pressure } \\
\quad \text { BP, mmHg) }\end{array}$ & $138 \pm 23 /$ & $137 \pm 20 /$ & $138 \pm 24 /$ & $141 \pm 24 /$ \\
$\begin{array}{c}\text { Prior surgery/ } \\
\text { immobility }\end{array}$ & $80 \pm 13$ & $79 \pm 13$ & $81 \pm 13$ & $83 \pm 9$ \\
$\begin{array}{c}\text { Autoimmune disease / } \\
\text { steroid therapy }\end{array}$ & $35(15)$ & $11(13)$ & $20(20)$ & $4(10)$ \\
$\begin{array}{c}\text { Pregnancy/hormonal } \\
\text { therapy }\end{array}$ & $15(7)$ & $5(6)$ & $7(7)$ & $3(7)$ \\
\hline
\end{tabular}

The aims of the study were to clarify patterns of resolution of perfusion defects after PE, and whether residual perfusion defects on $\mathrm{V} / \mathrm{P}$ SPECT indicate an increased risk of PE recurrence.

\section{Patients and methods}

Patients

We retrospectively followed all 227 patients diagnosed with PE who had undergone at least 2 examinations with V/P SPECT during 2005-2007 at Lund University Hospital, Lund, Sweden. The follow-up $\mathrm{V} / \mathrm{P}$ SPECT had been performed routinely in all these patients.

All patients were evaluated for risk factors for VTE such as malignancy, inflammatory bowel disease, smoking, pregnancy or hormonal therapy, surgery or immobilisation within the previous 3 months, and hereditary coagulation defects. Right ventricular strain was evaluated with echocardiography in 81 (36\%) patients, and 45 patients underwent MDCT in addition to V/P SPECT.

\section{V/P SPECT examinations}

PE was quantified by counting segments or subsegments showing complete or relative mismatch, and expressing this figure in \% of the total lung parenchyma [16]. A segmental reduction or a sub-segmental total deficiency of function was attributed 1 point, and segmental total deficiency 2 points. According to our charts each lung comprises 9 segments, representing 18 points. Mismatch defects were expressed as mismatch points, which after division by 36 give the fraction of the lung that is embolised. All regions with ventilation or perfusion defects were calculated in order to estimate the reduction in total lung function. However, quantification of PE is somewhat imprecise as one segment may contain one as well as two subsegments. Nevertheless quantification may provide a useful "rule of thumb" to define an initial treatment period which can then be confirmed using follow-up V/P SPECT.

Recurrent PE was defined as a new objectively confirmed diagnosis of symptomatic PE after the second V/P SPECT examination.

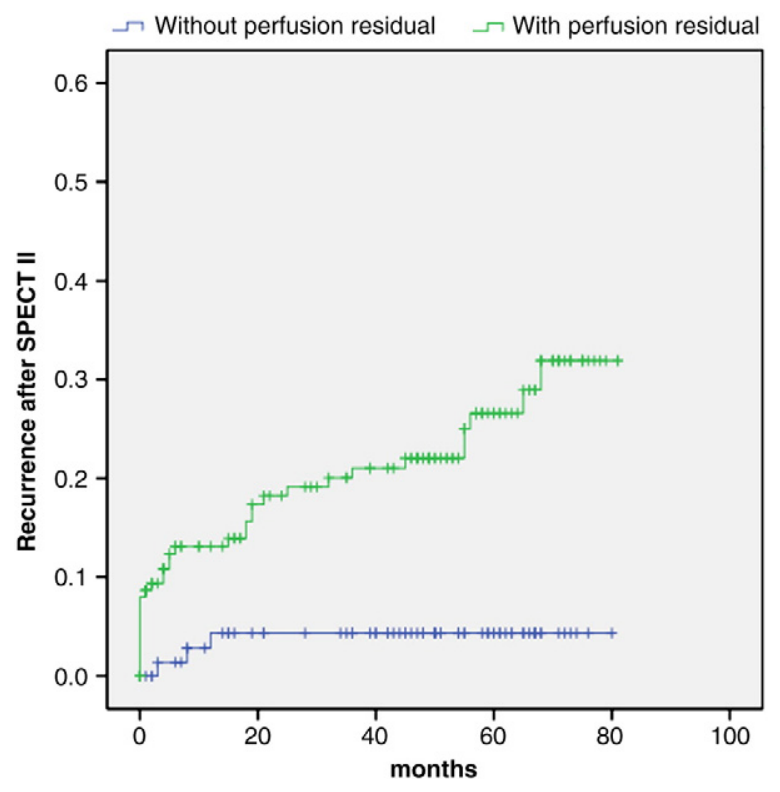

Fig. 1. Kaplan Meier curves showing recurrence of pulmonary embolism (PE) after second examination with ventilation / perfusion (VP) lung scintigraphy with tomographic (SPECT) technique in 227 patients with PE. The upper curve shows patients with residual perfusion defects and the lower curve those without.

\section{Statistics}

Results are given as mean \pm SD. Differences over time within groups were evaluated with Wilcoxon's signed rank test and differences between groups were evaluated with the Mann-Whitney U-test and the Chi-square test. Factors that differed between subjects with and without recurrent $\mathrm{PE}$ were entered into a multivariate regression analysis. P-values $<0.05$ were considered as significant. Kaplan-Meier Survival Analysis was used to estimate recurrence of PE during follow up.

The study was approved by the Ethical Committee at the University of Lund. Informed consent was obtained from all subjects.

\section{Results}

Patients were categorised according to PE extent: minor PE $(<20 \%$ perfusion defect on SPECT, $\mathrm{n}=86$ ), medium PE (20-50\% perfusion defect on SPECT, $n=99$ ), or major PE ( $>50 \%$ perfusion defect on SPECT, $\mathrm{n}=42$ ). Background data for the whole patient sample is shown in Table 1.

Risk factors for venous thromboembolism (VTE) were present in 79 (35\%) patients; prior VTE in 49 (22\%), and active malignancy in $51(24 \%)$. Hereditary coagulation defects were evaluated in 67 (30\%) patients, and could be documented in 22 (33\%) of these. Echocardiography was performed on 75 patients, of whom 39(17\%) showed right ventricular strain. All patients with PE were treated with anticoagulant therapy for $7.5 \pm 4.0$ months. Thrombolytic treatment was given in the acute stage to 16 patients with major PE aged $61 \pm 11$ years.

\section{Table 2}

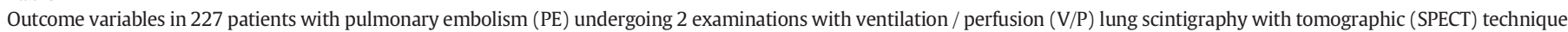

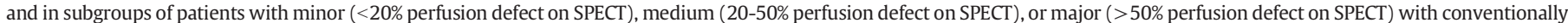
treated PE. Patients treated with thrombolysis are shown separately. Mean $\pm \mathrm{SD}$ or $\mathrm{n}(\%)$.

\begin{tabular}{|c|c|c|c|c|c|}
\hline & All patients $n=227$ & Minor PE $n=86$ & Medium PE $n=99$ & Major PE no thrombolysis, $\mathrm{n}=26$ & Major PE with thrombolysis, $\mathrm{n}=16$ \\
\hline Months of follow-up & $47 \pm 24$ & $47 \pm 24$ & $48 \pm 25$ & $48 \pm 24$ & $47 \pm 24$ \\
\hline Months to second V/P SPECT & $7.4 \pm 6.8$ & $8.2 \pm 7.4$ & $6.2 \pm 5.9$ & $6.5 \pm 0.7$ & \\
\hline Resolution of PE at second V/P SPECT & $76(33)$ & $45(52)$ & $29(30)$ & $2(5)$ & $0(0)$ \\
\hline Recurrent PE after second V/P SPECT & $37(16)$ & $7(8)$ & $23(23)$ & $5(19)$ & $2(12)$ \\
\hline Death & $59(26)$ & $17(20)$ & $33(33)$ & $9(21)$ & $0(0)$ \\
\hline
\end{tabular}


At the second V/P SPECT examination, complete resolution of perfusion defects had occurred in 45 (52\%) of patients with minor PE after $8.2 \pm 7.4$ months, in $29(29 \%)$ of patients with medium PE after $6.2 \pm 5.9$ months, and in 2(5\%) of patients with major PE after $6.5 \pm 0.7$ months (Table 2 ).

During $47 \pm 24$ months of follow up 59 patients (26\%) died and $37(16 \%)$ suffered recurrent PE after the second V/P SPECT examination (Table 2, Figs. 1-2). Only four of these 37 patients had ongoing therapeutic anticoagulation. Seventeen (20\%) of the deaths and 7 (8\%) of the recurrences occurred in the group with minor PE, 33 (33\%) of the deaths and 23 (23\%) of the recurrences in the group with medium PE, $9(21 \%)$ of the deaths, and $5(19 \%)$ of the recurrences in the group with major PE (Table 2).

Among the 37 patients experiencing recurrent $\mathrm{PE}$ after the second V/P SPECT examination, 34 (92\%) had exhibited residual perfusion defects at this investigation (Figs. 1-2). Recurrence of PE was also predicted by advanced age and female gender (Table 3 ). In multivariate regression analysis, however, recurrence of $\mathrm{PE}$ after the second $\mathrm{V} / \mathrm{P}$ SPECT examination was only predicted by age $(p=0.0013)$ and residual perfusion defect ( $p=0.0039$, Table 4 ). The extension of perfusion defect on V/P SPECT examination in 227 patients with PE at diagnosis, after 3, 6 and $>6$ months during ongoing anticoagulant therapy is shown in Fig. 3.

\section{Discussion}

We found that complete resolution of PE was common (52\%), especially in patients with minor PE, whereas residual perfusion defects were widespread in patients with medium and major PE;71\% and 95\% respectively after $6.5 \pm 0.7$ months.

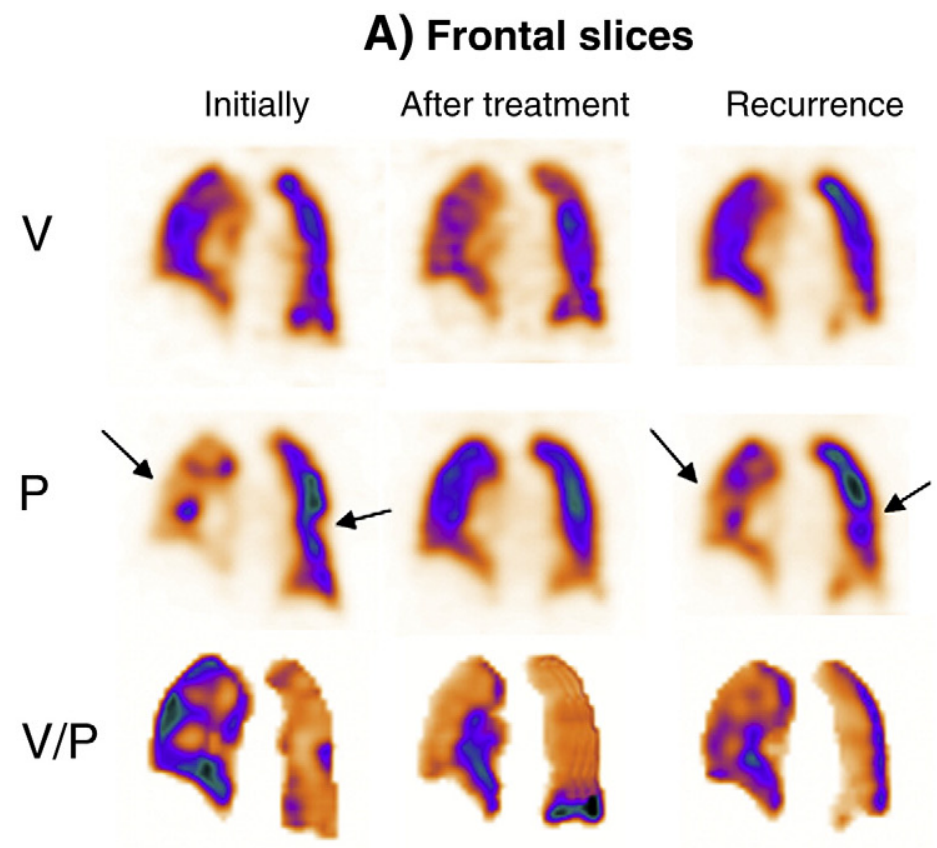

\section{B) Sagital slices, right lung}
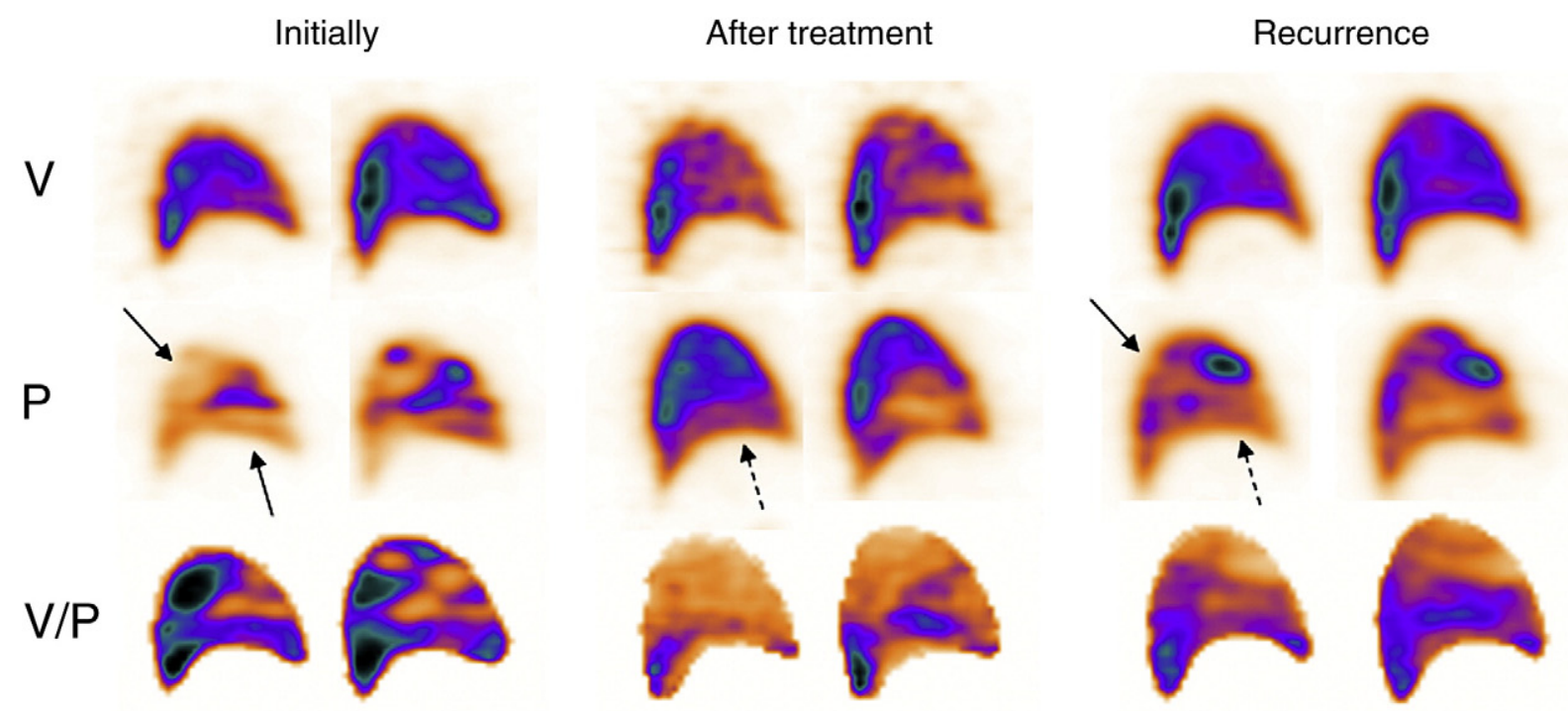

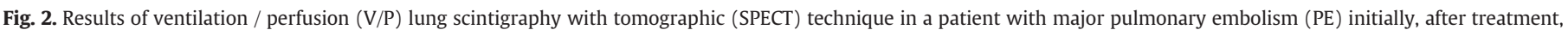
and at a later clinical recurrence of PE. Frontal (2a) and sagittal (2b) slices shown. 


\section{Table 3}

Factors predicting recurrence of pulmonary embolism (PE) after second examination with ventilation / perfusion (VP) lung scintigraphy with tomographic (SPECT) technique in 227 PE patients. Mean \pm SD or $\mathrm{n}(\%)$.

\begin{tabular}{llll}
\hline & $\begin{array}{l}\text { With } \\
\text { recurrence, } \\
\mathrm{n}=37\end{array}$ & $\begin{array}{l}\text { Without } \\
\text { recurrence, } \\
\mathrm{n}=190\end{array}$ & P-value \\
\hline Age (years) & $71 \pm 16$ & $62 \pm 17$ & $<0.0001$ \\
Gender M/F & $10 / 27$ & $84 / 106$ & 0.05 \\
Prior VTE (n[\%]) & $8(22)$ & $42(22)$ & 0.95 \\
Malignancy (n[\%]) & $7(19)$ & $44(23)$ & 0.63 \\
Blood pressure (BP, mmHg) & $136 \pm 20 /$ & $139 \pm 23 /$ & $0.59 /$ \\
& $81 \pm 11$ & $80 \pm 13$ & 0.14 \\
Residual perfusion defect & $34(92)$ & $117(62)$ & 0.0004 \\
$\quad$ at second SPECT & $1(3)$ & $23(12)$ & 0.088 \\
Prior surgery/immobility & $6(16)$ & $29(15)$ & 0.88 \\
Autoimmune disease /steroid therapy & $1(3)$ & $14(7)$ & 0.29 \\
Pregnancy/hormonal therapy & $21(57)$ & $121(64)$ & 0.43 \\
Completion of anticoagulant therapy at & & & \\
$\quad$ second SPECT & & &
\end{tabular}

Regarding minor PE, our results are in agreement with recently published data from a prospective study showing that already after 2 weeks of treatment, $43 \%$ patients with PE had normalised perfusion [16]. These findings are also consistent with earlier observations $[17,18]$. In the study by Begic et al. [16] patients were also examined after 3 and 6 months as these time intervals are often used for anticoagulant treatment after venous thromboembolism. Interestingly, on examination with V/P SPECT 70\% of patients in this study showed no perfusion defects after 3 months and patients who did not improve after 3 months showed a tendency to develop chronic PE [16]. Our results are consistent with these findings particularly in patients with minor PE. On the other hand, Wartsky et al. [19] showed higher prevalence of residual perfusion defects at 3 months. Miniati et al. [20] reported normalised perfusion in $65 \%$ of patients after 1 year in patients who survived extensive PE.

Patients with PE are currently recommended at least 6 months of anticoagulation treatment in current guidelines both from Europe and USA $[8,9,21]$. Whether a strategy of follow-up of PE patients with V/P SPECT and cessation of treatment after documented resolution of PE is feasible and safe needs to be evaluated in a properly randomised trial.

Some patients with major PE in this study were treated with thrombolysis, which facilitated early resolution of PE. Evaluation of PE resolution both in early and later stages after thrombolysis is another field in which V/P SPECT should be evaluated in a separate prospective setting. When evaluating risk for recurrent deep venous thrombosis (DVT), residual thrombosis detected by phlebography or ultrasound has been shown to be a prognostic factor, but only in patients without thrombosis predisposing factors [22-24].

In our study of patients with PE using repeated V/P SPECT, we had the opportunity to evaluate whether signs of residual PE were an indicator of possible PE recurrence and found that residual perfusion defects were present at the second SPECT examination in 34 (92\%) of the 37 patients experiencing recurrent $\mathrm{PE}$.

When evaluating factors affecting the risk of PE recurrence anticoagulation treatment must be taken into account. However, as

Table 4

Multivariate analysis of factors predicting recurrence of pulmonary embolism (PE) after second examination with ventilation / perfusion (VP) lung scintigraphy with tomographic (SPECT) technique in 227 PE patients. Mean \pm SD or $\mathrm{n}(\%)$.

\begin{tabular}{llrlr}
\hline & $\begin{array}{l}\text { Significance } \\
\text { (p value) }\end{array}$ & $\begin{array}{l}\text { Odds } \\
\text { ratio } \\
\text { (OR) }\end{array}$ & $\begin{array}{l}\text { 95\% CI for } \\
\text { or lower }\end{array}$ & $\begin{array}{l}\text { 95\% CI for } \\
\text { or upper }\end{array}$ \\
\hline Age (years) & 0.0013 & 1.083 & 1.031 & 1.137 \\
$\begin{array}{l}\text { Gender M/F } \\
\text { Residual perfusion defect at } \\
\text { second V/P SPECT }\end{array}$ & 0.1966 & 0.543 & 0.215 & 1.372 \\
\hline
\end{tabular}

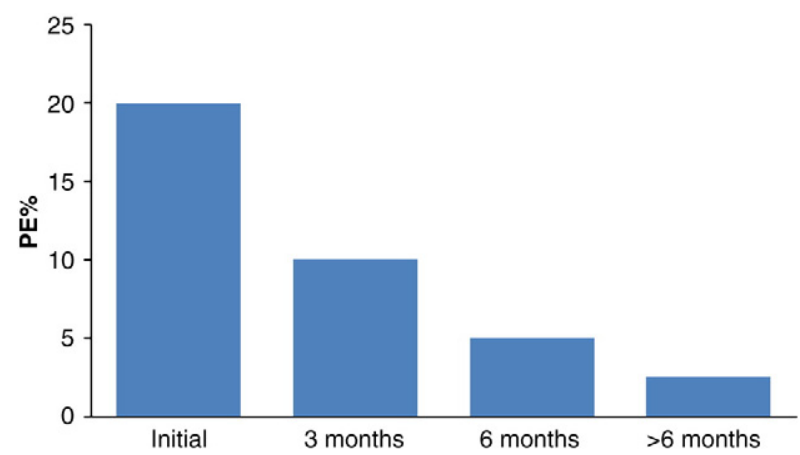

Fig. 3. The extension (\%) of perfusion defect on ventilation / perfusion (VP) lung scintigraphy with tomographic (SPECT) technique in 227 patients with pulmonary embolism (PE) at diagnosis, and after $3(n=117), 6(n=44)$ and $>6$ months $(n=66)$ during ongoing anticoagulant therapy.

relationships between anticoagulation and recurrence are complex and as this was not a controlled study, this variable is difficult to evaluate. As a precaution, clinicians may choose to recommend an extended duration of anticoagulation if a follow-up V/P SPECT shows residual perfusion defects. No formal guidelines on treatment duration in relation to $\mathrm{V} / \mathrm{P}$ SPECT results existed in our department at the time of the study, however, only four patients experienced recurrent PE during ongoing therapeutic anticoagulation.

Owing to its retrospective nature, this study has several other limitations; for example the fact that cardiopulmonary comorbidity was poorly documented might have complicated the analysis. None of the patients that underwent two V/P SPECT examinations were lost to follow-up, but the study did not include patients with PE who died before the second examination or patients who failed to attend the second examination. Although current clinical practice at the department during the study period included follow-up of PE with repeat V/P SPECT examinations, clinicians may also have judged follow-up unnecessary in some cases. A total of around $300 \mathrm{~V} / \mathrm{P}$ SPECT examinations on PE patients are performed yearly at the department, meaning that $227 \times 2=454$ of around $900 \mathrm{~V} / \mathrm{P} \mathrm{SPECT}$ examinations performed during the period have been studied. Our aims were therefore possible to evaluate in only about a third of all PE patients during the study period.

In conclusion, complete resolution of PE was common in patients with minor PE, whereas residual perfusion defects were widespread in patients with medium and major PE. PE patients with persistent perfusion defects on follow-up V/P SPECT have a high risk of PE recurrence.

\section{Conflict of interest statement}

No conflicts of interests exist for any of the authors of this study.

\section{Acknowledgements}

This study was supported by grants from the Ernhold Lundström Foundation, Research Funds at Skåne University Hospital, the Albert Påhlsson Foundation, the Hulda Ahlmroth Foundation, the Swedish state under the LUA/ALF agreement, the Swedish Heart and LungFoundation, and the Swedish Medical Research Council (grant 02872).

\section{References}

[1] Bajc M, Bitzén U, Olsson B, Perez de Sá V, Palmer J, Jonson B. Lung ventilation/perfusion SPECT in the artificially embolized pig. J Nucl Med 2002;43:640-7.

[2] Gutte H, Mortensen J, Jensen CV, von der Recke P, Petersen CL, Kristoffersen US, et al. Comparison of V/Q SPECT and planar V/Q lung scintigraphy in diagnosing acute pulmonary embolism. Nucl Med Commun 2010;31:82-6. 
[3] Reinartz P, Wildberger JE, Schaefer W, Nowak B, Mahnken AH, Buell U. Tomographic Imaging in the Diagnosis of Pulmonary Embolism: A Comparison Between V/Q Lung Scintigraphy in SPECT Technique and Multislice Spiral CT. J Nucl Med 2004;45: 1501-8.

[4] Bajc M, Neilly JB, Miniati M, Schuemichen C, Meignan M, Jonson B. EANM guidelines for ventilation/perfusion scintigraphy: Part 2. Algorithms and clinical considerations for diagnosis of pulmonary emboli with V/P(SPECT) and MDCT. Eur J Nucl Med Mol Imaging 2009;36:1528-38.

[5] ICRP. Radiation dose to patients from radiopharmaceuticals, publication 80 (addendum to ICRP 53). Ann ICRP 1998;28.

[6] Hurwitz LM, Yoshizumi TT, Goodman PC, Nelson RC, Toncheva G, Nguyen GB, et al Radiation dose savings for adult pulmonary embolus 64-MDCT using bismuth breast shields, lower peak kilovoltage, and automatic tube current modulation. AJR Am J Roentgenol 2009;192:244-53.

[7] Hurwitz LM, Yoshizumi TT, Reiman RE, Paulson EK, Frush DP, Nguyen GT, et al. Radiation dose to the female breast from 16-MDCT body protocols. AJR Am J Roentgenol 2006;186:1718-22.

[8] Kearon C, Kahn SR, Agnelli G, Goldhaber S, Raskob GE, Comerota A] Antithrombotic therapy for venous thromboembolic disease: American College of Chest Physicians Evidence-Based Clinical Practice Guidelines (8th Edition). Chest 2008;133:454S-545S.

[9] Torbicki A, Perrier A, Konstantinides S, Agnelli G, Galiè N, Pruszczyk P, et al. Guidelines on the diagnosis and management of acute pulmonary embolism: the Task Force for the Diagnosis and Management of Acute Pulmonary Embolism of the European Society of Cardiology (ESC). Eur Heart J 2008;29:2276-315.

[10] Kearon C. Stopping anticoagulant therapy after an unprovoked venous thromboembolism. CMAJ 2008;179:401-2.

[11] Hurwitz LM, Reiman RE, Yoshizumi TT, Goodman PC, Toncheva G, Nguyen G, et al. Radiation dose from contemporary cardiothoracic multidetector CT protocols with an anthropomorphic female phantom: implications for cancer induction. Radiology 2007;245:742-50.

[12] ICRP. Managing patient dose in multi-detector computed tomography (MDCT). Ann ICRP 2007; 102

[13] Tunariu N, Gibbs SJ, Win Z, Gin-Sing W, Graham A, Gishen P, et al. Ventilation-perfusion scintigraphy is more sensitive than multidetector CTPA in detecting chronic thromboembolic pulmonary disease as a treatable cause of pulmonary hypertension. J Nucl Med 2007;48:680-4.
[14] Soler X, Kerr KM, Marsh JJ, Renner JW, Hoh CK, Test VJ, et al. Pilot study comparing SPECT perfusion scintigraphy with CT pulmonary angiography in chronic thromboembolic pulmonary hypertension. Respirology 2012;180:1440-843.

[15] He J, Fang W, Lv B, He JG, Xiong CM, Liu ZH, et al. Diagnosis of chronic thromboembolic pulmonary hypertension: comparison of ventilation/perfusion scanning and multidetector computed tomography pulmonary angiography with pulmonary angiography. Nucl Med Commun 2012 May;33(5):459-63.

[16] Begic A, Jögi J, Hadziredzepovic A, Kucukalic-Selimović E, Begovic-Hadzimuratovic S, Bajc M. Tomographic ventilation/perfusion lung scintigraphy in the monitoring of the effect of treatment in pulmonary embolism: serial follow-up over a 6-month period. Nucl Med Commun 2011;32:508-14.

[17] Kearon C. Natural history of venous thromboembolism. Circulation 2003;107: I22-30.

[18] Goldhaber SZ, Haire WD, Feldstein ML, Miller M, Toltzis R, Smith JL, et al. Alteplase versus heparin in acute pulmonary embolism: randomised trial assessing right-ventricular function and pulmonary perfusion. Lancet 1993;341:507-11.

[19] Wartski M, Collignon MA. Incomplete recovery of lung perfusion after 3 months in patients with acute pulmonary embolism treated with antithrombotic agents. THESEE Study Group. Tinzaparin ou Heparin Standard: Evaluation dans l'Embolie Pulmonaire Study. J Nucl Med 2000;41:1043-8.

[20] Miniati M, Monti S, Bottai M, Scoscia E, Bauleo C, Tonelli L, et al. Survival and restoration of pulmonary perfusion in a long-term follow-up of patients after acute pulmonary embolism. Med (Baltimore) 2006;85:253-62.

[21] Segal JB, Streiff MB, Hofmann LV, Thornton K, Bass EB. Management of venous thromboembolism: a systematic review for a practice guideline. Ann Intern Med 2007:146:211-22

[22] Prandoni P, Prins MH, Lensing AW, Ghirarduzzi A, Ageno W, Imberti D, et al. AESOPUS Investigators. Residual thrombosis on ultrasonography to guide the duration of anticoagulation in patients with deep venous thrombosis: a randomized trial. Ann Intern Med 2009;150:577-85.

[23] Tan M, Mos IC, Klok FA, Huisman MV. Residual venous thrombosis as predictive factor for recurrent venous thromboembolim in patients with proximal deep vein thrombosis: a sytematic review. Br J Haematol 2011;153:168-78.

[24] Carrier M, Rodger MA, Wells PS, Righini M, LE Gal G. Residual vein obstruction to predict the risk of recurrent venous thromboembolism in patients with deep vein thrombosis: a systematic review and meta-analysis. J Thromb Haemost 2011;9: 1119-25. 